\title{
Immature platelets and antiplatelet therapy response to aspirin in Kawasaki disease
}

This article was published in the following Dove Press journal:

Drug Design, Development and Therapy

Lei $\mathrm{Pi},{ }^{1}, *$ Di Che, ${ }^{1, *}$ Haifeng Long, ${ }^{2,3, *}$ Zhenzhen Fang, ${ }^{4}$ Jiawen Li,' Shuyi Lin, ${ }^{2}$ Yunfeng Liu, ${ }^{2}$ Meiai Li, ${ }^{2}$ Lijuan Bao, ${ }^{2}$ Wenli Li, ${ }^{2}$ Yuan Zhang, ${ }^{2}$ Qiulian Deng, ${ }^{2}$ Techang Liu, ${ }^{5}$ Li Zhang, ${ }^{5}$ Xiaoqiong $\mathrm{Gu}^{1,2}$

'Department of Clinical Biological Resource Bank, Guangzhou Institute of Pediatrics, Guangzhou Women and Children's Medical Center, Guangzhou Medical University, Guangzhou, China; 2Department of Clinical Laboratory, Guangzhou Women and Children's Medical Center, Guangzhou Medical University, Guangzhou, China; ${ }^{3}$ Department of Clinical Laboratory, The First Clinical Medical College, Jinan University, Guangzhou, China; ${ }^{4}$ Program of Molecular Medicine, Guangzhou Women and Children's Hospital, Zhongshan School of Medicine, Sun Yat-Sen University, Guangzhou, China; ${ }^{5}$ Department of Cardiology, Guangzhou Women and Children's Medical Center, Guangzhou Medical University, Guangzhou, China

*These authors contributed equally to this work

Correspondence: Xiaoqiong Gu Department of Clinical Laboratory, Guangzhou Institute of Pediatrics, Guangzhou Women and Children's Medical Center, Guangzhou Medical University, 9 Jinsui Road, Guangzhou 510623, Guangdong, China

Tel +86203807 656

Fax +86203807 656I

Email guxiaoqiong@।63.com

Li Zhang

Department of Cardiology, Guangzhou Women and Children's Medical Center, Guangzhou Medical University, 9 Jinsui Road, Guangzhou

510623, Guangdong, China

Tel +86 2081330675

Fax +86 2081330675

Email zhangliheart2016@163.com
Introduction: Kawasaki disease is a kind of systemic vasculitis that mainly damages moderate and small-sized blood vessels, and is a leading cause of coronary artery lesions (CAL). Antiplatelet therapy is a routine component of Kawasaki disease treatment strategies. So it is important to evaluate the antiplatelet effect of aspirin because of the individual biological variability of antiplatelet effect of aspirin. The immature platelet fraction (IPF) has attracted particular attention as it may influence the antiplatelet effect of aspirin. This study investigated the prognostic factors for evaluating the degree of vasculitis and the effect of antiplatelet therapy in children with Kawasaki disease. Materials and methods: Blood samples were collected from 44 patients with Kawasaki disease before aspirin treatment and 7 to 10 days after treatment. The IPF counts, percentage of the IPF, and highly fluorescent IPF were detected by a Sysmex XE-5000 instrument. The levels of 11-dehydrothromboxane $\mathrm{B}_{2}\left(11-\mathrm{DH}-\mathrm{TXB}_{2}\right.$ ), soluble $\mathrm{CD} 40$ ligand (sCD40L), and soluble $\mathrm{P}$-selectin (sP-selectin) were measured by ELISA. The correlation between the measured factors and the degree of coronary artery damage in Kawasaki disease was analyzed.

Results: We found that 11-DH-TXB ${ }_{2}$, sP-selectin, and SCD40L levels were much more elevated in the CAL group than in the non-coronary artery lesions (NCAL) group before aspirin treatment. The concentrations of 11-DH-TXB ${ }_{2}, \mathrm{sCD} 40 \mathrm{~L}, \mathrm{sP}$-selectin, and IPF were reduced after aspirin treatment in the NCAL group but not the CAL group. This is related to the degree of coronary artery damage in Kawasaki disease patients. Additionally, 11-DH-TXB ${ }_{2}$, sCD40L, sP-selectin, and IPF were positively correlated with the degree of coronary artery damage in Kawasaki disease patients.

Conclusion: The current study suggests that the presence of high plasma concentrations of 11-DH-TXB ${ }_{2}$, sCD40L, sP-selectin, and IPF can be considered a risk factor and experimental biomarker for CAL in Kawasaki disease patients.

Keywords: Kawasaki disease, coronary artery lesions, aspirin, immature platelet fraction

\section{Introduction}

Kawasaki disease is a kind of systemic vasculitis that mainly damages moderate and smallsized blood vessels, especially the coronary artery, occurring mainly in children younger than 5 years old. ${ }^{1}$ The most serious complication of Kawasaki disease is coronary artery lesions (CAL), including coronary artery dilation, aneurysms, or fistula formation. Additionally, CAL is the leading cause of death in children with Kawasaki disease. ${ }^{2,3}$ Although Kawasaki disease is a self-limiting disease in most patients, there have been reports that up to $25 \%$ of Kawasaki disease patients will develop CAL if not given treatment with intravenous immunoglobulin (IVIG) and aspirin. Therefore, Kawasaki disease is recognized as a leading cause of acquired heart disease in children in developed countries. ${ }^{1}$

So far, the first line treatment for Kawasaki disease is high-dose IVIG in combination with oral aspirin. The acute phase of Kawasaki disease is thought to involve 
platelet activation; therefore, antiplatelet therapy is a routine component of Kawasaki disease treatment strategies. ${ }^{4,5}$ Aspirin has been administered in Kawasaki disease treatment for decades. Low-dose aspirin (3-5 mg/kg/day) is recommended to prevent platelet activation and aggregation. ${ }^{6,7}$ The antiplatelet effects of aspirin for healing endothelial dysfunction and preventing clot formation are necessary for decreasing the occurrence of coronary artery complications. However, the antiplatelet effect of aspirin displays considerable individual biological variability, which probably results from several different mechanisms. ${ }^{8,9}$ So it is necessary to evaluate the antiplatelet effect of aspirin.

Reticulated platelets represent a fraction of immature circulating platelets that are newly released from the bone marrow and whose larger surface area and increased granules and nucleic acid content has been linked to higher activation. ${ }^{10}$ The immature platelet fraction (IPF) is a parameter that similarly represents platelet turnover in the bloodstream; displays a good correlation with the rate of reticulated platelets; and allows for a more reproducible, cheaper, and precise measurement. ${ }^{11,12}$ In the last decade, IPF has attracted particular attention because of its influence on the antiplatelet effect of aspirin. ${ }^{13,14}$ A higher level of IPF means a larger amount of immature, reticulated platelets with increased hemostatic potential in the blood. ${ }^{14}$ Previous studies have revealed the relationship between IPF with premature coronary artery disease, acute cardiovascular events, and impaired response to antiplatelet agents. ${ }^{15-17}$ 11-dehydrothromboxane $\mathrm{B}_{2}\left(11-\mathrm{DH}-\mathrm{TXB}_{2}\right)$, the end-product of the $\mathrm{TXA}_{2}$ pathway, has been regarded as a marker of aspirin activity and cited as one of the most reliable means for assessing an individual's response to aspirin. Furthermore, both circulating soluble P-selectin (sP-selectin) and soluble CD40 ligand (sCD40L) expression are recognized as markers of platelet activation and storage lesion.

Therefore, the goal of the present study was to compare these markers in Kawasaki disease patients with CAL to those without CAL, and to demonstrate the value of them in evaluating the degree of vasculitis and the effect of antiplatelet therapy in Kawasaki disease. Moreover, this study demonstrated the mechanism by which increased IPF, 11-DH-TXB ${ }_{2}$, sP-selectin, and sCD40L decreased the antiplatelet effect of aspirin during therapy.

\section{Ethics approval and consent to participate}

This study was performed with the approval of the Institutional Committee of Guangzhou Women and Children's Medical Center (2014073009). All the participants gave written informed consent.

\section{Materials and methods Study population}

We enrolled patients with Kawasaki disease from Guangzhou Women and Children's Medical Center in China. The study group included 44 Kawasaki disease patients (29 males and 15 females), all of whom met the criteria proposed by the Japanese Kawasaki Disease Research Committee. ${ }^{12}$ These patients were treated with oral aspirin and 1 or $2 \mathrm{~g} / \mathrm{kg}$ of IVIG after admission. All IVIG non-responders were excluded from the research. The dose of aspirin was $30-50 \mathrm{mg} \mathrm{kg}^{-1}$ day $^{-1}$ when the patients had a fever and changed to $3-5 \mathrm{mg} \mathrm{kg}^{-1}$ day $^{-1}$ when the patients became afebrile. In addition, 23 sex- and age-matched healthy children were used as healthy controls. Written consent to participate in the study was obtained from all the patients' guardians as their representatives. Echocardiography was obtained within 2 weeks of the disease onset. CAL were defined as coronary vessels with an internal diameter $\geq 3.0 \mathrm{~mm}$ in a child aged younger than 5 years or greater than $4.0 \mathrm{~mm}$ in those aged 5 years and older, using Japanese diagnostic guidelines. ${ }^{1}$ Patients were divided into two groups according to the presence of CAL: 21 patients with CAL and 23 patients without CAL. According to the coronary vessels' internal diameter, patients with CAL were divided into small coronary artery aneurysm $(<5.0 \mathrm{~mm})$, middle coronary artery aneurysm $(5.0-8.0 \mathrm{~mm})$, and large coronary artery aneurysm $(>8.0 \mathrm{~mm}) .{ }^{1}$ This study obtained the approval from the Ethics Committee of the Guangzhou Women and Children's Medical Center. All participants gave written informed consent for this study.

\section{Sample collection and clinical examination}

For each patient, $2 \mathrm{~mL}$ of venous blood was collected into collection tubes containing Dipotassium EDTA $(1.5 \mathrm{mg} / \mathrm{mL})$ before and 7 to 14 days after aspirin treatment. Total blood cell counts were analyzed within $2 \mathrm{~h}$ of sampling by an automatic blood cell counter (Sysmex XE-5000, Sysmex, Kobe, Japan). Absolute immature platelet fraction counts $\left(\mathrm{IPF}^{\#}\right)$ were obtained, the percentage of immature platelet fraction (IPF\%) was calculated as the percentage of IPF ${ }^{\#}$ to the total platelet counts (PLTs) (H-IPF represents the percentage of highly fluorescent immature platelet fraction to the total PLTs), and other platelet related parameters were established by a fully automated sorting system (forward light scatter vs fluorescence scatter plot) using the Sysmex XE-5000 instrument. Then, the blood samples were centrifuged at 2,500 rcf for $10 \mathrm{~min}$ to yield the platelets' free plasma, which was stored at $-80^{\circ} \mathrm{C}$ until the concentrations of 11-DH-TXB, sCD40L, and sP-selectin were assayed by 
ELISA. For the healthy controls, blood samples were collected only once.

\section{Biochemical measurements}

Concentrations of 11-DH-TXB ${ }_{2}$, SCD40L, and sP-selectin were determined in the plasma by commercially available quantitative ELISA according to the manufacturer's instructions. In brief, $40 \mu \mathrm{L}$ of plasma was used for each of the 11-DH-TXB ${ }_{2}, \mathrm{sCD} 40 \mathrm{~L}$, and sP-selectin measurements. All the samples were assayed in duplicate. After addition of the sample or standard, in every 11-DH-TXB ${ }_{2}, \mathrm{sCD} 40 \mathrm{~L}$, and sP-selectin plate, $50 \mu \mathrm{L}$ of horseradish peroxidase conjugated antibody was added to all the wells, and the plates were incubated at room temperature for $1 \mathrm{~h}$. After the incubation, the wells were washed five times with approximately $400 \mu \mathrm{L}$ of wash buffer. After all the liquid was removed, $100 \mu \mathrm{L}$ of tetramethylbenzidine solution was added to each well for color development, and the plates were incubated in the dark at room temperature for $30 \mathrm{~min}$. When the color development was complete, $50 \mu \mathrm{L}$ of stop solution was added to each well, and the plate was read within $15 \mathrm{~min}$ on a spectrophotometer using $450 \mathrm{~nm}$ as the primary wavelength. The mean optical densities were converted to concentrations using a standard curve run for each individual plate.

\section{Statistical analyses}

Statistical analysis was performed with SPSS software for Windows (Statistical Package for Social Sciences [IBM Corporation, Armonk, NY, USA] Version 22.0). All the values are presented as the mean $\pm \mathrm{SD}$ or as the frequency (percentage). The Wilcoxon rank sum test was used to test for differences between the Kawasaki disease cases and the healthy controls for continuous variables, and the Pearson $\chi^{2}$ test was used to test for differences between the groups for categorical variables. Paired $t$-tests were used to test for differences in the percentage of IPF before and after aspirin treatment.

\section{Results}

\section{Demographic characteristics of the patients studied}

During the study, 44 Kawasaki disease patients from the Guangzhou Women and Children's Medical Center were successfully recruited for participation in the study and had the appropriate blood work done (Table 1). According to the two-dimensional echocardiography, 21 patients were assigned to the CAL group and 23 to the non-coronary artery lesions (NCAL) group. During the same timeframe,
Table I Demographic data of healthy controls and patients with Kawasaki disease

\begin{tabular}{lllll}
\hline Variables & HC & NCAL & CAL & $P$-value \\
\hline $\begin{array}{l}\text { Age range, } \\
\text { months }\end{array}$ & $6-66$ & $7-84$ & $1-125$ & \\
$\begin{array}{l}\text { Mean } \pm \text { SD } \\
\text { Sex }\end{array}$ & $28.21 \pm 18.49$ & $30.47 \pm 19.40$ & $33.04 \pm 29.97$ & NS \\
$\quad$ Male & 16 & 17 & 12 & NS \\
$\quad$ Female & 7 & 6 & 9 & \\
MPV (FL) & $8.80 \pm 0.55$ & $10.16 \pm 1.96^{*}$ & $11.20 \pm 9.4^{*}$ & 0.00 \\
PDW (\%) & $0.36 \pm 0.08$ & $0.40 \pm 0.10$ & $0.40 \pm 0.13$ & NS \\
PCT (\%) & $11.96 \pm 1.42$ & $9.53 \pm 0.94$ & $10.45 \pm 1.02$ & NS \\
\hline
\end{tabular}

Notes: $P$-value was calculated by one-way ANOVA test. *Indicates a significant difference $(P<0.05)$ by comparison of controls.

Abbreviations: HC, healthy control; NCAL, non-coronary artery lesions; CAL, coronary artery lesions; MPV, mean platelet volume; PDW, platelet distribution width; PCT, plateletcrit; NS, not significant.

23 healthy individuals were recruited as the control group. The mean age and sex were similar for both groups.

\section{Changes in PLT, IPF", H-IPF, and IPF\% in each group of Kawasaki disease patients before aspirin treatment}

The PLT was not significantly different in the NCAL group or the CAL group compared with the healthy controls $(364.95 \pm 73.31) \times 10^{9} / \mathrm{L},(338.67 \pm 68.24) \times 10^{9} / \mathrm{L}$ vs $(334.04 \pm 56.79) \times 10^{9} / \mathrm{L}$ (Figure 1A). As shown in Figure 1B, the $\mathrm{IPF}^{\#}$ of the CAL group and the NCAL group were significantly higher than that of the healthy control group (7.15 \pm 2.87 vs $6.7 \pm 2.37$ and $4.06 \pm 1.65$ ). Moreover, H-IPF of the CAL group and the NCAL group were significantly higher than that of the healthy control group $(0.72 \pm 0.574$ vs $0.55 \pm 0.346$ and $0.32 \pm 0.09$ ) (Figure 1C). As shown in Figure 1D, the IPF\% of the CAL group and the NCAL group were significantly higher than that of the healthy control group ( $1.85 \pm 0.89$ vs $1.89 \pm 0.74$ and $1.04 \pm 0.42)$; however, there was no significant difference in the mean side fluorescence intensity of platelet (PLT-X) between the CAL and NCAL groups compared with the healthy control group $(15.07 \pm 1.53$ vs $14.54 \pm 1.27$ and $15.11 \pm 4.72)$ (Figure 1E).

\section{Plasma concentrations of I I-DH-TXB , sCD40L, and sP-selectin for each group of Kawasaki disease patients before aspirin treatment}

The plasma concentration of 11-DH-TXB ${ }_{2}$ in the CAL group was significantly higher than that in the NCAL group and the healthy control group $(2,041.73 \pm 401.94$ vs $1,601.96 \pm 386.59 \mathrm{ng} / \mathrm{mL}$ and $572.64 \pm 338.21 \mathrm{ng} / \mathrm{mL}$ ) (Figure 2A). As shown in Figure 2B, the sCD40L was also 


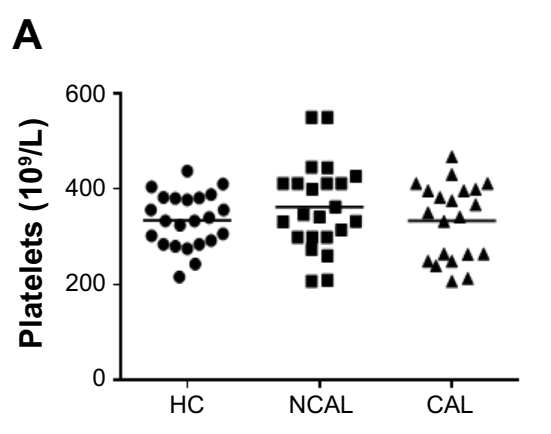

D

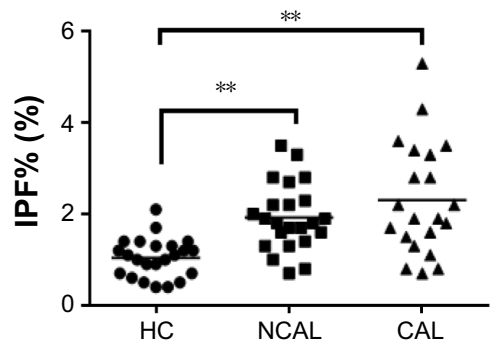

B

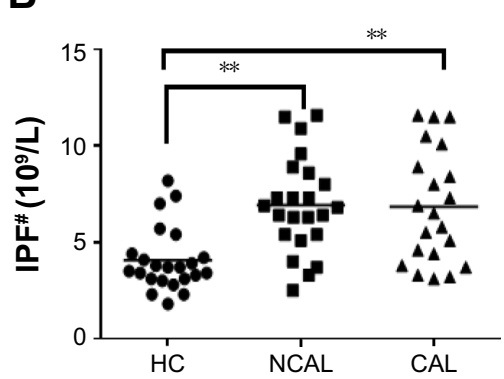

C

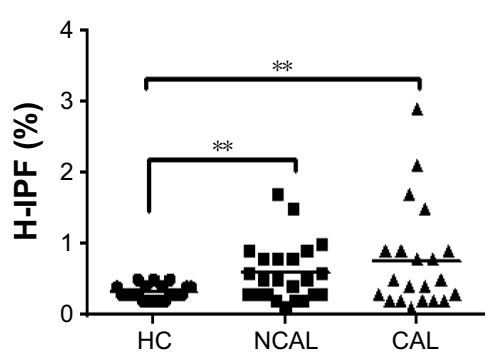

E

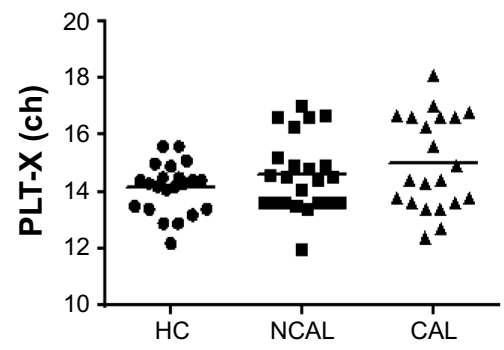

Figure I Changes in the platelet related results (counts, IPF", H-IPF, IPF\%, and PLT-X) in each group of Kawasaki disease patients before aspirin treatment. Notes: (A) The levels of PLT, (B) the levels of IPF", (C) the levels of H-IPF, (D) the levels of IPF\%, and (E) the levels of PLT-X. P $<0.05$ was considered statistically significant. $* * P<0.01, C A L$ group vs healthy control group.

Abbreviations: IPF", immature platelet fraction counts; H-IPF, percentage of highly fluorescent immature platelet fraction to the total platelet count; IPF\%, percentage of immature platelet fraction; PLT-X, mean side fluorescence intensity of platelet; PLT, platelet count; HC, healthy control; NCAL, non-coronary artery lesions; CAL, coronary artery lesions; ch, channel.

significantly higher in the CAL group than in NCAL group and the healthy control group $(21.11 \pm 2.68 \mathrm{vs} 17.78 \pm 3.24 \mathrm{ng} / \mathrm{mL}$ and $6.72 \pm 4.43 \mathrm{ng} / \mathrm{mL}$ ), while the sP-selectin expression was also significantly higher in the CAL group than that of the NCAL and healthy control group (25.48 \pm 3.97 vs 19.07 $\pm 3.44 \mathrm{ng} / \mathrm{mL}$ and $2.47 \pm 1.01 \mathrm{ng} / \mathrm{mL}$ ) (Figure 2C).

\section{The variation of IPF", H-IPF, and IPF\% in each group of Kawasaki disease patients after aspirin treatment}

The IPF related parameters were measured before and after aspirin treatment. In total, the patients without CAL undergoing aspirin treatment experienced a notable decrease. The IPF" and $\mathrm{H}-\mathrm{IPF}$ were significantly higher in the NCAL group than in the healthy control group (6.7 \pm 2.37 vs $4.06 \pm 1.64$ and $0.55 \pm 0.34$ vs $0.32 \pm 0.09)$ and was significantly lower in the NCAL group after aspirin treatment than before aspirin treatment $(4.88 \pm 1.94$ vs $6.7 \pm 2.37$ and $0.34 \pm 0.22$ vs $0.55 \pm 0.34$ ) (Figure $3 A$ and D); however, there was no significant difference in the IPF" and H-IPF between the CAL group after aspirin treatment and before aspirin treatment $(6.74 \pm 2.85$ vs $7.15 \pm 2.87$ and $0.48 \pm 0.34$ vs $0.72 \pm 0.57$ ) (Figure $3 \mathrm{~B}$ and E). Furthermore, the IPF\% was significantly higher in the NCAL group than that of the healthy control group (1.89 \pm 0.74 vs $1.04 \pm 0.42)$ and lower
A

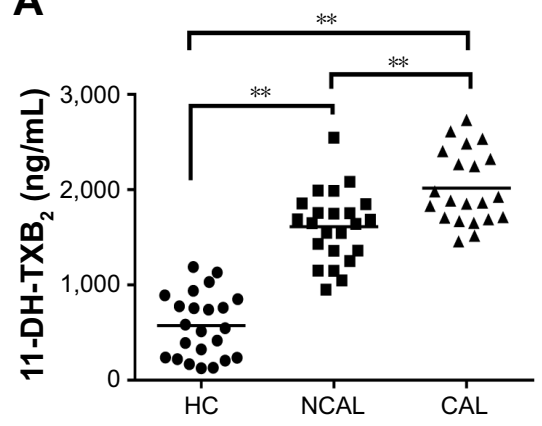

B

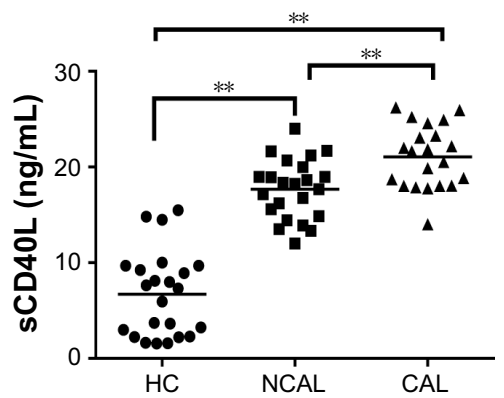

C

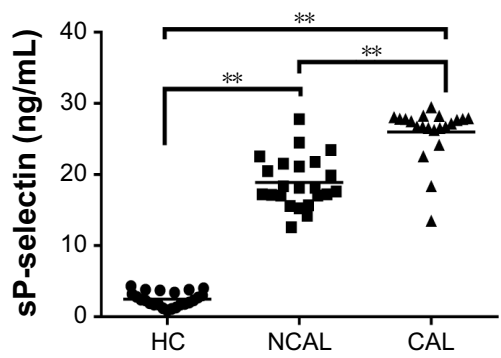

Figure 2 Plasma concentrations of II-DH-TXB, $\mathrm{sCD} 40 \mathrm{~L}$, and sP-selectin in each group of Kawasaki disease patients before aspirin treatment and in healthy controls. Notes: (A) Plasma concentration of II-DH-TXB ${ }_{2},(\mathbf{B})$ plasma concentration of $s \mathrm{CD} 40 \mathrm{~L}$, and $(\mathbf{C})$ plasma concentration of sP-selectin. $\mathrm{P}<0.05$ was considered statistically significant. $* * P<0.0$ I, NCAL group vs CAL group.

Abbreviations: II-DH-TXB ${ }_{2}$, I I-dehydrothromboxane $\mathrm{B}_{2}$; sCD40L, soluble CD40 ligand; sP-selectin, soluble P-selectin; HC, healthy control; NCAL, non-coronary artery lesions; CAL, coronary artery lesions. 
A

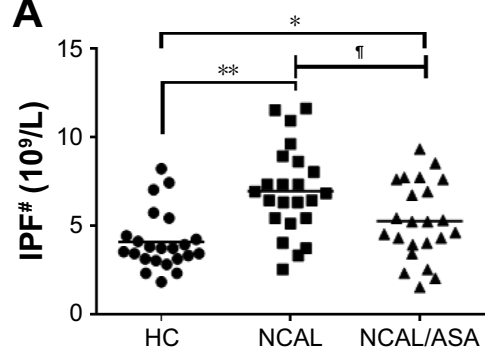

D

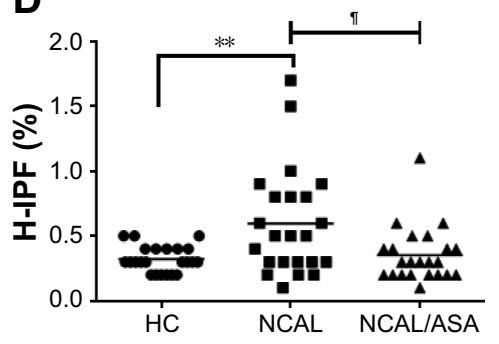

G

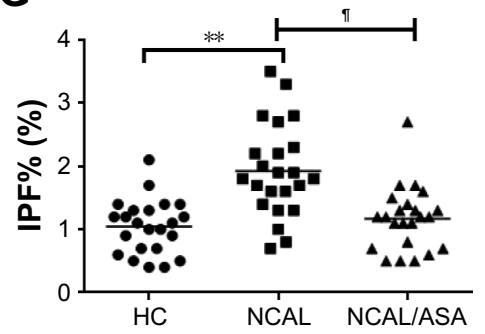

B

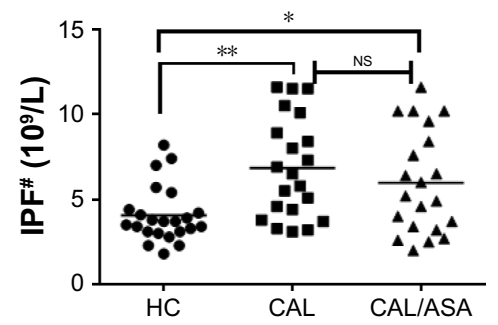

E

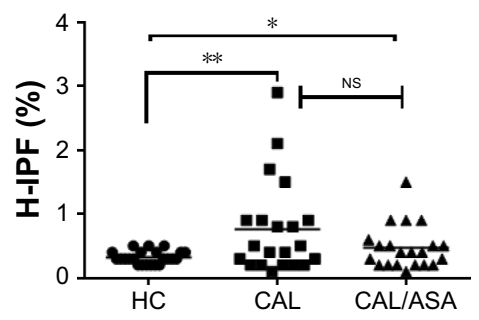

H

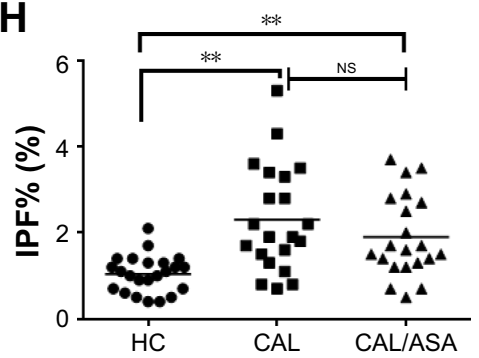

C

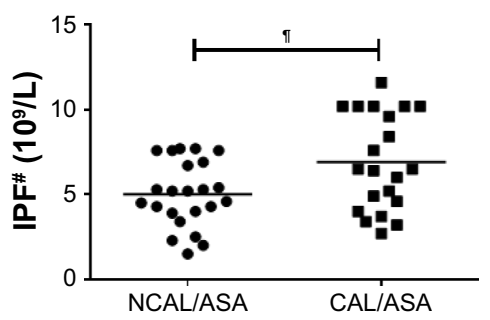

$\mathbf{F}$

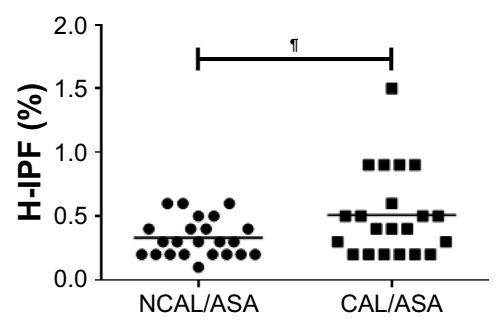

I

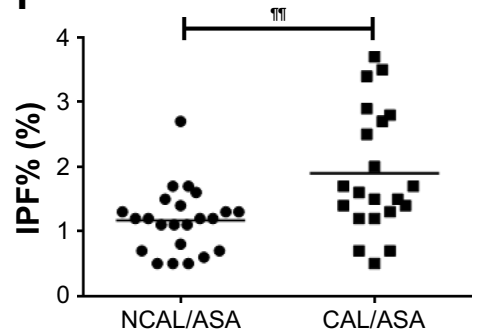

Figure 3 The variations of IPF", H-IPF, and IPF\% in each group of Kawasaki disease patients before and after aspirin treatment.

Notes: (A) The levels of IPF" before and after aspirin treatment in the NCAL group, (B) the levels of IPF" before and after aspirin treatment in the CAL group, (C) the levels of IPF after aspirin treatment in NCAL and CAL group, (D) the levels of H-IPF before and after aspirin treatment in the NCAL group, (E) levels of H-IPF before and after aspirin treatment in the CAL group, (F) levels of H-IPF after aspirin treatment in NCAL and CAL group, (G) levels of IPF\% before and after aspirin treatment in the NCAL group, (H) levels of IPF\% before and after aspirin treatment in the CAL group, (I) levels of IPF\% after aspirin treatment in NCAL and CAL group. $P<0.05$ was considered

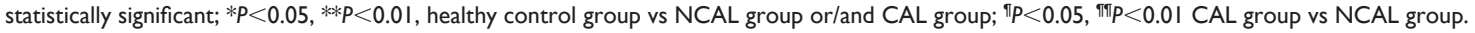

Abbreviations: IPF , immature platelet fraction counts; H-IPF, percentage of highly fluorescent immature platelet fraction to the total platelet count; IPF\%, percentage of immature platelet fraction; NCAL, non-coronary artery lesions; CAL, coronary artery lesions; HC, healthy control; NCAL/ASA, NCAL group after aspirin treatment; CAL/ASA, CAL group after aspirin treatment; NS, not significant.

in the NCAL group after aspirin treatment than before aspirin treatment (1.14 \pm 0.51 vs $1.89 \pm 0.74)$ (Figure $3 \mathrm{G}$ ). Additionally, there was no significant difference in $\mathrm{IPF} \%$ between the CAL group after aspirin treatment compared to before aspirin treatment (1.85 \pm 0.89 vs $2.14 \pm 1.06$ ) (Figure $3 \mathrm{H}$ ). There was no significant difference in the PLT between the NCAL group and the CAL group after aspirin treatment (data not shown). The levels of IPF", H-IPF, and IPF\% were significantly higher in the CAL group than in the NCAL group after aspirin treatment $(6.74 \pm 2.85$ vs $4.88 \pm 1.94,0.48 \pm 0.34$ vs $0.34 \pm 0.22$, and $1.85 \pm 0.89$ vs $1.14 \pm 0.52$ ) (Figure $3 \mathrm{C}, \mathrm{F}$ and I).

\section{Plasma concentrations of I I-DH-TXB ${ }_{2}$, sCD40L, and sP-selectin for each group after aspirin treatment}

As indices of platelet activation and the thrombogenesis state, we observed the expression of sCD40L and sP-selectin in Kawasaki disease patients. Among patients with CAL, there was no significant difference in the plasma concentrations of 11-DH-TXB ${ }_{2}$ and $\mathrm{SCD} 40 \mathrm{~L}$ in patients whose treatment with aspirin was longer than 7 to 14 days compared with before aspirin treatment $(1,845.59 \pm 358.15$ vs $2,041.73 \pm 401.94 \mathrm{ng} / \mathrm{mL}$ and $21.11 \pm 2.67 \mathrm{vs} 21.02 \pm 3.29 \mathrm{ng} / \mathrm{mL})$ (Figure 4B and E). However, the concentrations of 11-DH$\mathrm{TXB}_{2}$ and $\mathrm{sCD} 40 \mathrm{~L}$ in the patients with NCAL who were treated with aspirin were significantly lower than before aspirin treatment $(1,217.01 \pm 398.69$ vs $1,601.96 \pm 386.58 \mathrm{ng} / \mathrm{mL}$ and $13.50 \pm 3.486$ vs $17.78 \pm 3.23 \mathrm{ng} / \mathrm{mL}$ ) (Figure 4A and D). The levels of 11-DH-TXB 2 and $\mathrm{sCD} 40 \mathrm{~L}$ were significantly higher in the patients with CAL than in the patients with NCAL after aspirin treatment $(1,845.59 \pm 358.15$ vs $1,217.01 \pm 398.69 \mathrm{ng} / \mathrm{mL}$ and $21.02 \pm 3.29 \mathrm{vs} 13.5 \pm 3.48 \mathrm{ng} / \mathrm{mL})$ (Figure 4C and F). Furthermore, the concentration of sP-selectin in the CAL and NCAL groups who were treated with aspirin was significantly lower than before aspirin treatment $(25.48 \pm 3.97 \mathrm{vs} 22.93 \pm 5.16 \mathrm{ng} / \mathrm{mL}$ and $19.07 \pm 3.44$ 
A

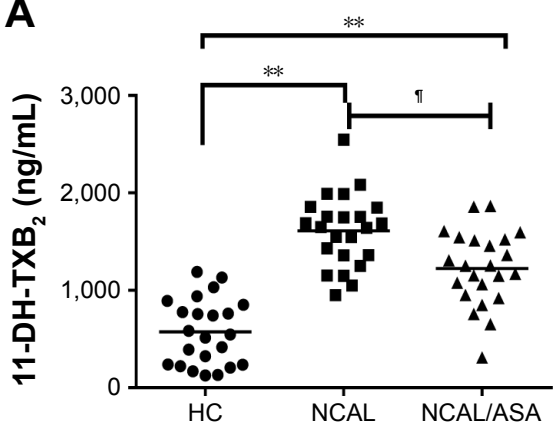

D

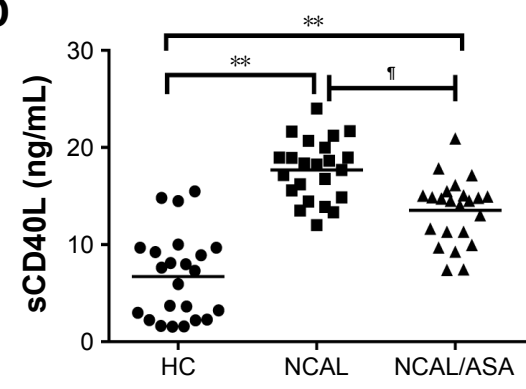

$\mathbf{G}$

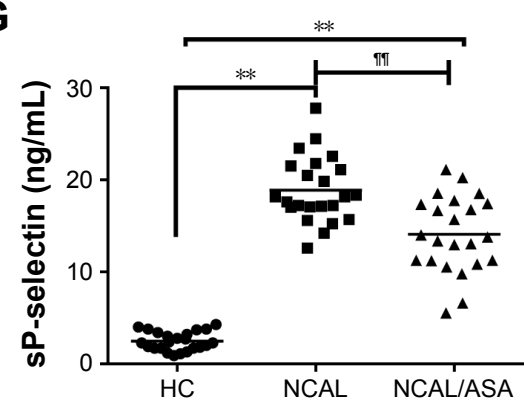

B

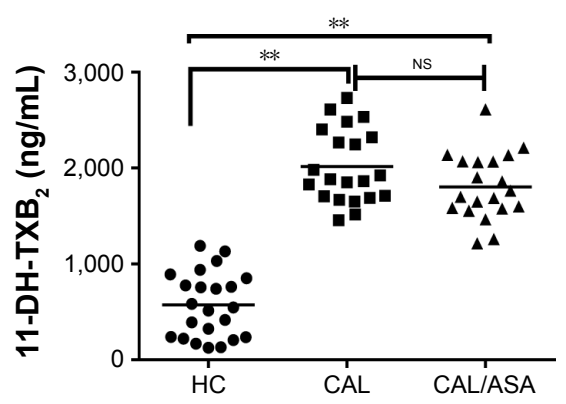

E

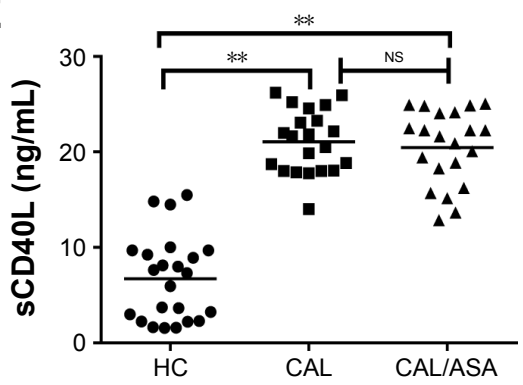

H

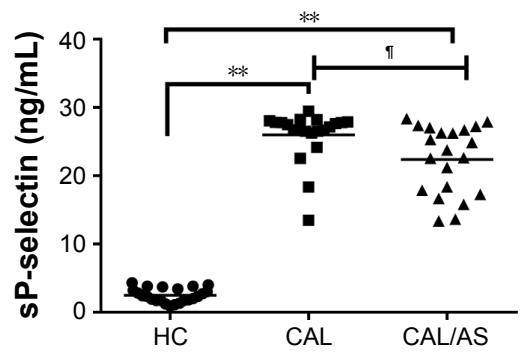

C

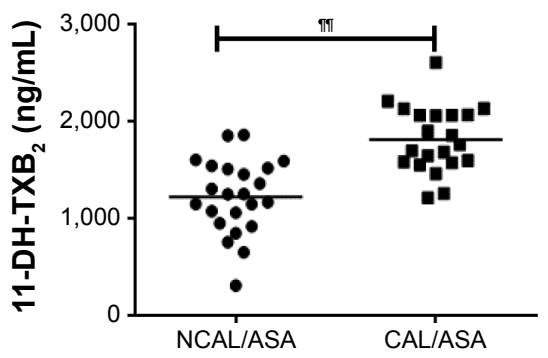

$\mathbf{F}$

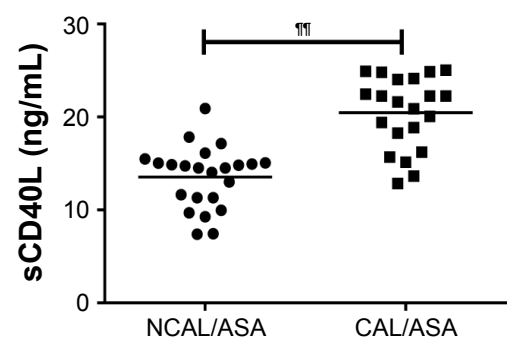

I

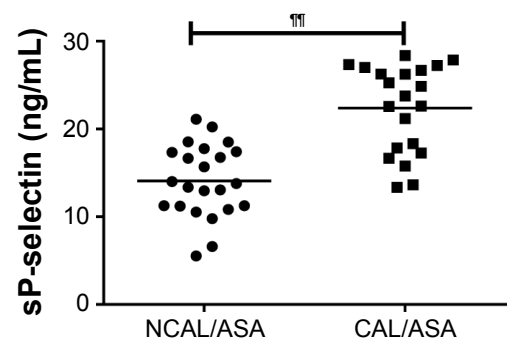

Figure 4 Plasma concentrations of II-DH-TXB, sCD40L, and sP-selectin in Kawasaki disease patients before and after aspirin treatment.

Notes: (A) Plasma concentration of II-DH-TXB ${ }_{2}$ before and after aspirin treatment in the NCAL group, (B) plasma concentration of II-DH-TXB in the CAL group, (C) plasma concentration of II-DH-TXB after aspirin treatment in NCAL and CAL group, (D) plasma concentration of sCD40L before and after aspirin treatment in the NCAL group, (E) plasma concentration of sCD40L in the CAL group, (F) plasma concentration of sCD40L after aspirin treatment in NCAL and CAL group, (G) plasma concentration of sP-selectin before and after aspirin treatment in the NCAL group, $(\mathbf{H})$ plasma concentration of sP-selectin in the CAL group, $(\mathbf{I})$ plasma concentration of sP-selectin after aspirin treatment in NCAL and CAL group. $P<0.05$ was considered statistically significant; $* * P<0.01$, healthy control group vs $N C A L$ group or/and $C A L$ group; $\pi P<0.05$, $\pi T P<0.01$, NCAL group vs $C A L$ group.

Abbreviations: II-DH-TXB ${ }_{2}$, I -dehydrothromboxane $\mathrm{B}_{2}$; sCD40L, soluble CD40 ligand; sP-selectin, soluble P-selectin; HC, healthy control; NCAL, non-coronary artery lesions; CAL, coronary artery lesions; NCAL/ASA, NCAL group after aspirin treatment; CAL/ASA, CAL group after aspirin treatment; NS, not significant.

vs $14.51 \pm 3.86 \mathrm{ng} / \mathrm{mL}$ ) (Figure $4 \mathrm{G}$ and $\mathrm{H}$ ), but the levels of sP-selectin were also significantly higher in the patients with CAL than in the patients without CAL after aspirin treatment (22.93 \pm 5.16 vs $14.51 \pm 3.86 \mathrm{ng} / \mathrm{mL})$ (Figure 4I).

\section{Relationship between the plasma} concentrations of I I-DH-TXB, sCD40L, sP-selectin, immature platelets factors, and the degree of coronary artery damage in Kawasaki disease patients

There was a positive correlation between the plasma 11-DH$\mathrm{TXB}_{2}, \mathrm{sCD} 40 \mathrm{~L}$, and sP-selectin concentration and the degree of coronary artery damage in Kawasaki disease patients (r=0.382, $P<0.01 ; \mathrm{r}=0.541, P<0.01 ; \mathrm{r}=0.551, P<0.01)$ (Figure 5A-C). Furthermore, the IPF and H-IPF were positively correlated with the degree of coronary artery damage in Kawasaki disease patients $(\mathrm{r}=0.447, P<0.01$; $\mathrm{r}=0.508$, $P<0.01$ ) (Figure 5D and E), but there was no obvious correlation between the IPF\% and the degree of coronary artery damage ( $\mathrm{r}=0.31, P=0.54)$ (Figure $5 \mathrm{~F})$. Multivariate regression showed that 11-DH-TXB, sCD40L, sP-selectin, IPF\%, and $\mathrm{H}-\mathrm{IPF}$ are significantly positively correlated with the degree of coronary artery damage $(P<0.05)$ (Table 2$)$.

\section{Discussion}

In this study, our data showed that the plasma concentrations of 11-DH-TXB ${ }_{2}, \mathrm{sCD} 40 \mathrm{~L}$, and sP-selectin in the CAL 

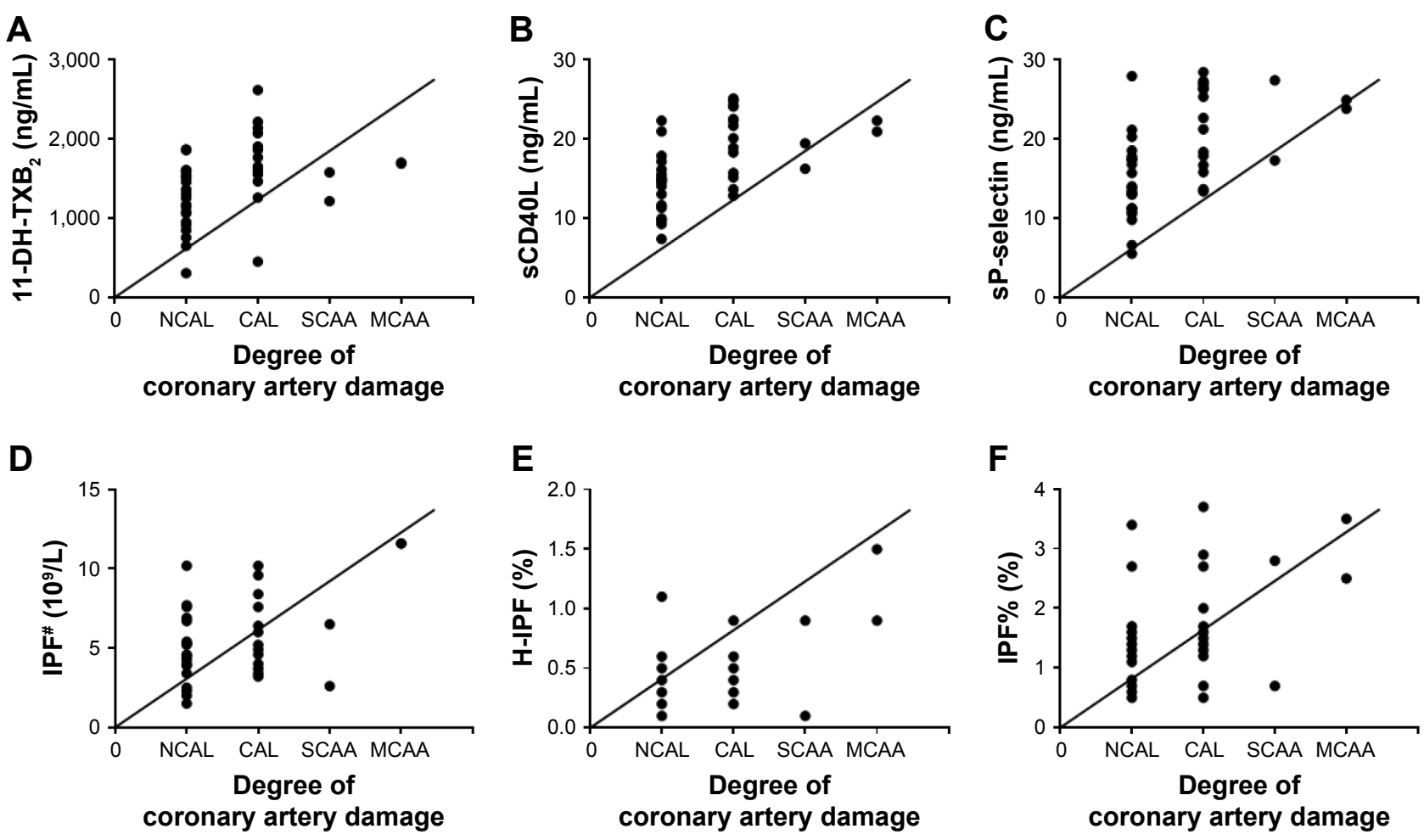

Figure 5 Relationship between the concentrations of II-DH-TXB ${ }_{2}$ sCD40L, sP-selectin, and IPF related results and the degree of coronary artery damage in Kawasaki disease patients.

Notes: Correlation between degree of coronary artery damage and the plasma concentrations of II-DH-TXB $(\mathbf{A})$, sCD40L (B), and sP-selectin (C) in Kawasaki disease patients. Correlation between degree of coronary artery damage and the levels of IPF ${ }^{\#}$ (D), H-IPF (E), and IPF\% (F) in Kawasaki disease patients. The Kawasaki disease patients were divided into four subgroups according to the degree of coronary artery damage: NCAL $(n=23)$; CAL $(n=17)$; SCAA ( $n=2)$; MCAA ( $n=2)$.

Abbreviations: II-DH-TXB , II-dehydrothromboxane $\mathrm{B}_{2}$; sCD40L, soluble CD40 ligand; sP-selectin, soluble P-selectin; IPF, immature platelet fraction; IPF", immature platelet fraction counts; H-IPF, percentage of highly fluorescent immature platelet fraction to the total platelet count; IPF\%, percentage of immature platelet fraction; NCAL, non-coronary artery lesions; CAL, coronary artery lesions; SCAA, small coronary artery aneurysm; MCAA, medium coronary artery aneurysm.

group were significantly higher than that of the NCAL group. Furthermore, the levels of IPF ${ }^{\#}, \mathrm{H}-\mathrm{IPF}$, and IPF\% were also significantly higher in the CAL group than in the NCAL group after aspirin treatment, which positively correlates with the degree of coronary artery damage in Kawasaki disease patients after low-dose aspirin treatment. In Kawasaki disease patients, elevated 11-DH-TXB, sCD40L, and IPF

Table 2 Multivariate regression analysis of the plasma concentration of II-DH-TXB, sCD40L, IPF, H-IPF, IPF\% and degree of coronary artery damage in Kawasaki disease patients

\begin{tabular}{lllll}
\hline Variables & $\mathbf{R}$ & $\mathbf{S E}$ & $\mathbf{9 5 \%} \mathbf{C l}$ & P-value \\
\hline II-DH-TXB $(\mathrm{ng} / \mathrm{mL})$ & 0.039 & 0.113 & $0.178,0.629$ & 0.013 \\
sCD40L $(\mathrm{ng} / \mathrm{mL})$ & 0.556 & 0.077 & $0.394,0.705$ & 0.000 \\
sP-selectin $(\mathrm{ng} / \mathrm{mL})$ & 0.535 & 0.094 & $0.346,0.716$ & 0.000 \\
IPF\% $(\%)$ & 0.445 & 0.174 & $0.043,0.738$ & 0.004 \\
H-IPF $(\%)$ & 0.483 & 0.240 & $0.115,0.814$ & 0.002 \\
IPF $\left(\times 10^{9} / \mathrm{L}\right)$ & 0.310 & 0.190 & $0.101,0.620$ & 0.054 \\
PLT-X (ch) & 0.252 & 0.200 & $0.198,0.585$ & 0.121 \\
\hline
\end{tabular}

Abbreviations: II-DH-TXB, II-dehydrothromboxane $\mathrm{B}_{2}$; sCD40L, soluble CD40 ligand; sP-selectin, soluble P-selectin; IPF, immature platelet fraction; H-IPF, percentage of highly fluorescent immature platelet fraction to the total platelet count; IPF\%, percentage of immature platelet fraction; IPF", immature platelet fraction counts; PLT-X, mean side fluorescence intensity of platelet. levels before aspirin treatment, which cannot be reduced by antiplatelet therapy, indicate an increased risk of developing CAL.

$11-\mathrm{DH}-\mathrm{TXB}_{2}$ is the metabolite of $\mathrm{TXB}_{2}$, and the plasma level of 11-DH-TXB 2 has been suggested to be an indicator

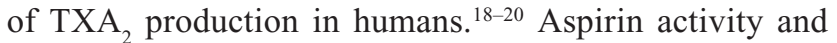
antiplatelet action can be evaluated by measurement of the end-products of the TXA 2 pathway, such as 11-DH-TXB ${ }_{2}$. The 11-DH-TXB ${ }_{2}$ levels also reflect the amount of $\mathrm{TXA}_{2}$ derived from other sources besides platelets, such as macrophages and monocytes. ${ }^{21}$ Santilli et al demonstrated a dose-dependent effect of aspirin on the 11-DH-TXB ${ }_{2}$ levels in patients with stable coronary artery disease. ${ }^{22}$ Chaudhary et al found that statin with high-dose aspirin therapy was associated with a reduction in urinary 11-DH-TXB ${ }_{2}$ levels in diabetic patients, indicating that urinary $11-\mathrm{DH}-\mathrm{TXB}_{2}$ levels may be a marker to determine the anti-inflammatory effect for therapy. ${ }^{23}$ In the present study, we found that the plasma 11-DH-TXB ${ }_{2}$ concentration in the CAL group was significantly higher than that of the NCAL group. Moreover, it was significantly higher in patients with CAL than 
in patients with NCAL after aspirin treatment. Meanwhile, it positively correlates with the degree of coronary artery damage in Kawasaki disease patients. It suggests that the presence of high plasma 11-DH-TXB ${ }_{2}$ concentration can be considered a risk factor and experimental biomarker for CAL in Kawasaki disease patients.

The immature platelets retain the megakaryocyte-derived RNA and the molecular machinery necessary for translation, as such, they are capable of augmenting protein synthesis on platelet activation, ${ }^{24}$ and are more capable of collagen-induced aggregation and thrombin generation when compared with older counterparts. ${ }^{25-28}$ Moreover, immature platelets have a greater propensity for thrombus formation. ${ }^{29}$ Accumulating evidence revealed that Kawasaki disease is associated with a higher IPF compared with that of healthy controls, which is higher in Kawasaki disease patients with CAL than in those without CAL. Another principal observation of this study is that the aspirin treatment of Kawasaki disease patients resulted in a reduction of the counts and percentages of IPF in the NCAL group, but not in normalization. These combined observations suggest that high IPF is caused, at least in part, by Kawasaki disease, which reflects the high platelet turnover. H-IPF represents the percentage of highly fluorescent platelets with a major amount of m-RNA within the IPF, which are the more active ones in immature platelets, and may be a useful marker of platelet activity. Reticulated platelets represent the fraction of immature circulating platelets whose larger surface area and increased granule content make more of a contribution to the secretion of sP-selectin and circulating sCD40L. Therefore, the increased counts and percentages of IPF could be explained, at least in part, by the higher level of sP-selectin and circulating sCD40L in Kawasaki disease patients.

P-selectin (CD62P, GMP-140), an adhesive glycoprotein integral to the membrane of alpha granules of platelets and the Weibel-Palade bodies of endothelial cells, has long been seen as a marker of platelet secretion and activation in vitro. It is mobilized to the cell surface upon alpha granule exocytosis and cleaved to generate sP-selectin, which mediates several early processes in inflammatory cell adhesion and induces a procoagulant state. ${ }^{30} \mathrm{CD} 40$ ligand is a trimeric transmembrane protein, a member of the tumor necrosis factor family, which is found in a variety of cell types, including endothelial cells, smooth muscle cells, monocytes, and macrophages; however, it is predominantly released into the blood stream from the activated platelet membrane surface as a soluble form, called sCD40L. ${ }^{31,32}$ Circulating sP-selectin and $\mathrm{SCD} 40 \mathrm{~L}$ are believed to be derived predominantly from activated platelets and hence may reflect the platelet activation condition. ${ }^{33,34}$ As such, they are considered biomarkers of platelet activation that have been associated with cardiovascular events. ${ }^{31,34,35}$ In the present study, we found that the sCD40L and sP-selectin levels were much more elevated in the CAL group than those in the NCAL group. Furthermore, the concentrations of sP-selectin and $\mathrm{SCD} 40 \mathrm{~L}$ in the NCAL group were significantly lower after aspirin treatment. However, the levels of sCD40L were also significantly higher in patients with CAL after aspirin treatment. Meanwhile, the level of sCD40L positively correlates with the degree of coronary artery damage in Kawasaki disease patients. This suggests that the presence of high plasma SCD40L concentration can be considered a risk factor and experimental biomarker for CAL in Kawasaki disease patients.

Some limitations of the present study must be acknowledged. First, the sample size in the current study is still not large enough. Multicenter studies with larger sample sizes are needed to verify the correlation between plasma 11-DH-TXB ${ }_{2}, \mathrm{sCD} 40 \mathrm{~L}$, and sP-selectin concentration, parameters relative to IPF, and the degree of coronary artery damage in Kawasaki disease.

\section{Conclusion}

In summary, the results of the current study provide evidence that levels of 11-DH-TXB ${ }_{2}$, sP-selectin, and SCD40L were much more elevated in the CAL group than those in the NCAL group of Kawasaki disease patients. Additionally, our evidence indicates that higher plasma 11-DH-TXB ${ }_{2}$, $\mathrm{sP}$-selectin, and $\mathrm{SCD} 40 \mathrm{~L}$ concentrations after aspirin treatment in Kawasaki disease patients are related to the degree of coronary artery damage in these patients. The elevated 11-DH-TXB ${ }_{2}$ and SCD40L levels and counts and percentages of IPF, which cannot be reduced by antiplatelet therapy, indicate an increased risk for developing CAL in Kawasaki disease patients. In conclusion, the presence of high plasma levels of 11-DH-TXB ${ }_{2}, \mathrm{sCD} 40 \mathrm{~L}$, sP-selectin, and IPF can be considered risk factors and biomarkers for CAL in Kawasaki disease patients.

\section{Acknowledgments}

The Clinical Biological Resource Bank of Guangzhou Women and Children's Medical Center is acknowledged for providing all the clinical samples. This study was supported by the National Key Basic Research and Development Program (973 Program), China, grant number 2015CB755402-037; Guangdong Natural Science Fund, China, grant number 2016A030313836; Guangzhou Science and Technology 
Program Project, China, grant numbers 201510010159, 201510010287, and 201607010011; Guangdong Science and Technology Project, China, grant numbers 2014A020212012, 2014A020212613, and 2014A020212023; Guangzhou medical and health technology projects, China, grant numbers 20171A011260, 20161A010030.

\section{Author contributions}

LP and DC carried out the molecular laboratory work, participated in data analysis, carried out sequence alignments, participated in the design of the study and drafted the manuscript. HFL, ML, LJB, and ZZF carried out the statistical analyses; JWL and SYL collected field data; YZ, QLD, TCL, and LZ conceived the study and designed the study. XQG coordinated the study and helped draft the manuscript. All authors contributed toward data analysis, drafting and critically revising the paper and agree to be accountable for all aspects of the work.

\section{Disclosure}

The authors report no conflicts of interest.

\section{References}

1. Newburger JW, Takahashi M, Gerber MA, et al. Diagnosis, treatment, and long-term management of Kawasaki disease: a statement for health professionals from the Committee on Rheumatic Fever, Endocarditis and Kawasaki Disease, Council on Cardiovascular Disease in the Young, American Heart Association. Circulation. 2004;110(17):2747-2771.

2. Kuo HC, Yang KD, Chang WC, Ger LP, Hsieh KS. Kawasaki disease: an update on diagnosis and treatment. Pediatr Neonatol. 2012;53(1):4-11.

3. Bayers S, Shulman ST, Paller AS. Kawasaki disease: part II. Complications and treatment. J Am Acad Dermatol. 2013;69(4):513. e511-e518.

4. Hamada H. [Aspirin treatment for patients with Kawasaki disease]. Nihon Rinsho. 2014;72(9):1612-1616. Japanese.

5. Sohn S, Kwon K. Accelerated thrombotic occlusion of a medium-sized coronary aneurysm in Kawasaki disease by the inhibitory effect of ibuprofen on aspirin. Pediatr Cardiol. 2008;29(1):153-156.

6. Kusakawa S, Tatara K. Efficacies and risks of aspirin in the treatment of the Kawasaki disease. Prog Clin Biol Res. 1987;250:401-413.

7. Kuo HC, Lo MH, Hsieh KS, Guo MM, Huang YH. High-dose aspirin is associated with anemia and does not confer benefit to disease outcomes in Kawasaki disease. PLoS One. 2015;10(12):e0144603.

8. Maree AO, Jneid H, Fitzgerald DJ. Aspirin resistance and atherothrombotic disease. J Am Coll Cardiol. 2006;48(4):846-847.

9. Grove EL, Kristensen SD. Update on oral antiplatelet therapy: principles, problems and promises. Future Cardiol. 2009;5(3):247-258.

10. Ault KA, Rinder HM, Mitchell J, et al. The significance of platelets with increased RNA content (reticulated platelets). A measure of the rate of thrombopoiesis. Am J Clin Pathol. 1992;98(6):637-646.

11. Briggs C, Harrison P, Machin SJ. Continuing developments with the automated platelet count. Int J Lab Hematol. 2007;29(2):77-91.

12. Pons I, Monteagudo M, Lucchetti G, et al. Correlation between immature platelet fraction and reticulated platelets. Usefulness in the etiology diagnosis of thrombocytopenia. Eur J Haematol. 2010;85(2):158-163.

13. Guthikonda S, Alviar CL, Vaduganathan M, et al. Role of reticulated platelets and platelet size heterogeneity on platelet activity after dual antiplatelet therapy with aspirin and clopidogrel in patients with stable coronary artery disease. J Am Coll Cardiol. 2008;52(9):743-749.
14. Guthikonda S, Lev EI, Patel R, et al. Reticulated platelets and uninhibited COX-1 and COX-2 decrease the antiplatelet effects of aspirin. J Thromb Haemost. 2007;5(3):490-496.

15. Cesari F, Marcucci R, Gori AM, et al. Reticulated platelets predict cardiovascular death in acute coronary syndrome patients. Insights from the AMI-Florence 2 Study. Thromb Haemost. 2013;109(5):846-853.

16. Ibrahim H, Nadipalli S, DeLao T, Guthikonda S, Kleiman NS. Immature platelet fraction (IPF) determined with an automated method predicts clopidogrel hyporesponsiveness. J Thromb Thrombolysis. 2012;33(2): 137-142.

17. Grove EL, Hvas AM, Kristensen SD. Immature platelets in patients with acute coronary syndromes. Thromb Haemost. 2009;101(1):151-156.

18. Kawamura M, Harada Y, Katori M. Evaluation of plasma 11-dehydrothromboxane B2 as an indicator for thromboxane A2 synthesis in vivo in laboratory animals. Thromb Res. 1995;77(5):465-474.

19. Catella F, Healy D, Lawson JA, FitzGerald GA. 11-Dehydrothromboxane B2: a quantitative index of thromboxane A2 formation in the human circulation. Proc Natl Acad Sci U S A. 1986;83(16):5861-5865.

20. Patrono C. Measurement of thromboxane biosynthesis in man. Eicosanoids. 1989;2(4):249-251.

21. DiChiara J, Bliden KP, Tantry US, et al. The effect of aspirin dosing on platelet function in diabetic and nondiabetic patients: an analysis from the aspirin-induced platelet effect (ASPECT) study. Diabetes. 2007; 56(12):3014-3019.

22. Santilli F, Paloscia L, Liani R, et al. Circulating myeloid-related protein$8 / 14$ is related to thromboxane-dependent platelet activation in patients with acute coronary syndrome, with and without ongoing low-dose aspirin treatment. J Am Heart Assoc. 2014;3(4). pii: e000903.

23. Chaudhary R, Bliden KP, Garg J, et al. Statin therapy and inflammation in patients with diabetes treated with high dose aspirin. J Diabetes Complications. 2016;30(7):1365-1370.

24. Eshel D, Toporik A, Efrati T, Nakav S, Chen A, Douvdevani A. Characterization of natural human antagonistic soluble CD40 isoforms produced through alternative splicing. Mol Immunol. 2008;46(2):250-257.

25. Esposito P, Rampino T, Dal Canton A. Soluble CD40 as a modulator of CD40 pathway. Immunol Lett. 2012;147(1-2):85-86.

26. Reyes-Moreno C, Girouard J, Lapointe R, Darveau A, Mourad W. CD40/ $\mathrm{CD} 40$ homodimers are required for CD40-induced phosphatidylinositol 3-kinase-dependent expression of B7.2 by human B lymphocytes. J Biol Chem. 2004;279(9):7799-7806.

27. Blumberg N, Spinelli SL, Francis CW, Taubman MB, Phipps RP. The platelet as an immune cell-CD40 ligand and transfusion immunomodulation. Immunol Res. 2009;45(2-3):251-260.

28. Pullen SS, Labadia ME, Ingraham RH, et al. High-affinity interactions of tumor necrosis factor receptor-associated factors (TRAFs) and CD40 require TRAF trimerization and CD40 multimerization. Biochemistry. 1999;38(31):10168-10177.

29. Ma DY, Clark EA. The role of CD 40 and CD154/CD40L in dendritic cells. Semin Immunol. 2009;21(5):265-272.

30. Burger PC, Wagner DD. Platelet P-selectin facilitates atherosclerotic lesion development. Blood. 2003;101(7):2661-2666.

31. Antoniades C, Bakogiannis C, Tousoulis D, Antonopoulos AS, Stefanadis C. The CD40/CD40 ligand system: linking inflammation with atherothrombosis. J Am Coll Cardiol. 2009;54(8):669-677.

32. Heeschen C, Dimmeler S, Hamm CW, et al. Soluble CD40 ligand in acute coronary syndromes. $N$ Engl J Med. 2003;348(12):1104-1111.

33. Chakrabarti S, Varghese S, Vitseva O, Tanriverdi K, Freedman JE. CD40 ligand influences platelet release of reactive oxygen intermediates. Arterioscler Thromb Vasc Biol. 2005;25(11):2428-2434.

34. Blann AD, Lip GY. Hypothesis: is soluble P-selectin a new marker of platelet activation? Atherosclerosis. 1997;128(2):135-138.

35. Sharma G, Berger JS. Platelet activity and cardiovascular risk in apparently healthy individuals: a review of the data. J Thromb Thrombolysis. 2011;32(2):201-208. 


\section{Publish your work in this journal}

Drug Design, Development and Therapy is an international, peerreviewed open-access journal that spans the spectrum of drug design and development through to clinical applications. Clinical outcomes, patient safety, and programs for the development and effective, safe, and sustained use of medicines are the features of the journal, which

has also been accepted for indexing on PubMed Central. The manuscript management system is completely online and includes a very quick and fair peer-review system, which is all easy to use. Visit http://www.dovepress.com/testimonials.php to read real quotes from published authors.

Submit your manuscript here: http://www.dovepress.com/drug-design-development-and-therapy-journal 